\title{
An Empirical Study on the Effect of Negation Words on Sentiment
}

\author{
Xiaodan Zhu, Hongyu Guo, Saif Mohammad and Svetlana Kiritchenko \\ National Research Council Canada \\ 1200 Montreal Road \\ Ottawa, K1A 0R6, ON, Canada \\ \{Xiaodan. Zhu, Hongyu.Guo, Saif.Mohammad, Svetlana.Kiritchenko\} \\ anrc-cnrc.gc.ca
}

\begin{abstract}
Negation words, such as no and not, play a fundamental role in modifying sentiment of textual expressions. We will refer to a negation word as the negator and the text span within the scope of the negator as the argument. Commonly used heuristics to estimate the sentiment of negated expressions rely simply on the sentiment of argument (and not on the negator or the argument itself). We use a sentiment treebank to show that these existing heuristics are poor estimators of sentiment. We then modify these heuristics to be dependent on the negators and show that this improves prediction. Next, we evaluate a recently proposed composition model (Socher et al., 2013) that relies on both the negator and the argument. This model learns the syntax and semantics of the negator's argument with a recursive neural network. We show that this approach performs better than those mentioned above. In addition, we explicitly incorporate the prior sentiment of the argument and observe that this information can help reduce fitting errors.
\end{abstract}

\section{Introduction}

Morante and Sporleder (2012) define negation to be "a grammatical category that allows the changing of the truth value of a proposition". Negation is often expressed through the use of negative signals or negators-words like isn't and never, and it can significantly affect the sentiment of its scope. Understanding the impact of negation on sentiment is essential in automatic analysis of sentiment. The literature contains interesting research attempting to model and understand the behavior (reviewed in Section 2). For example,

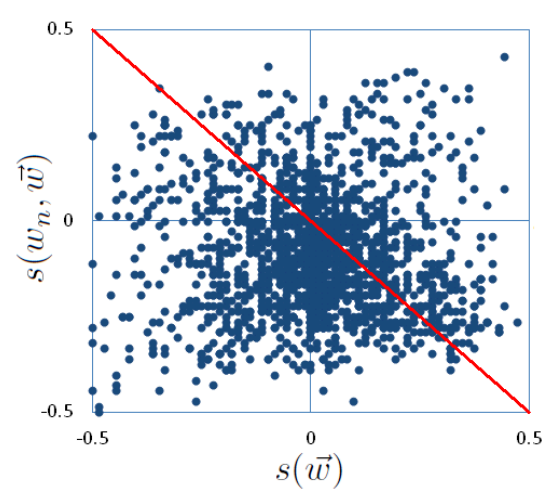

Figure 1: Effect of a list of common negators in modifying sentiment values in Stanford Sentiment Treebank. The x-axis is $s(\vec{w})$, and y-axis is $s\left(w_{n}, \vec{w}\right)$. Each dot in the figure corresponds to a text span being modified by (composed with) a negator in the treebank. The red diagonal line corresponds to the sentiment-reversing hypothesis that simply reverses the sign of sentiment values.

a simple yet influential hypothesis posits that a negator reverses the sign of the sentiment value of the modified text (Polanyi and Zaenen, 2004; Kennedy and Inkpen, 2006). The shifting hypothesis (Taboada et al., 2011), however, assumes that negators change sentiment values by a constant amount. In this paper, we refer to a negation word as the negator (e.g., isn't), a text span being modified by and composed with a negator as the argument (e.g., very good), and entire phrase (e.g., isn't very good) as the negated phrase.

The recently available Stanford Sentiment Treebank (Socher et al., 2013) renders manually annotated, real-valued sentiment scores for all phrases in parse trees. This corpus provides us with the data to further understand the quantitative behavior of negators, as the effect of negators can now be studied with arguments of rich syntactic and semantic variety. Figure 1 illustrates the effect of a common list of negators on sentiment as observed 
on the Stanford Sentiment Treebank. ${ }^{1}$ Each dot in the figure corresponds to a negated phrase in the treebank. The $\mathrm{x}$-axis is the sentiment score of its argument $s(\vec{w})$ and y-axis the sentiment score of the entire negated phrase $s\left(w_{n}, \vec{w}\right)$.

We can see that the reversing assumption (the red diagonal line) does capture some regularity of human perception, but rather roughly. Moreover, the figure shows that same or similar $s(\vec{w})$ scores (x-axis) can correspond to very different $s\left(w_{n}, \vec{w}\right)$ scores (y-axis), which, to some degree, suggests the potentially complicated behavior of negators. ${ }^{2}$

This paper describes a quantitative study of the effect of a list of frequent negators on sentiment. We regard the negators' behavior as an underlying function embedded in annotated data; we aim to model this function from different aspects. By examining sentiment compositions of negators and arguments, we model the quantitative behavior of negators in changing sentiment. That is, given a negated phrase (e.g., isn't very good) and the sentiment score of its argument (e.g., $s$ ("very good") $=0.5$ ), we focus on understanding the negator's quantitative behavior in yielding the sentiment score of the negated phrase $s$ ("isn't very good").

We first evaluate the modeling capabilities of two influential heuristics and show that they capture only very limited regularity of negators' effect. We then extend the models to be dependent on the negators and demonstrate that such a simple extension can significantly improve the performance of fitting to the human annotated data. Next, we evaluate a recently proposed composition model (Socher, 2013) that relies on both the negator and the argument. This model learns the syntax and semantics of the negator's argument with a recursive neural network. This approach performs significantly better than those mentioned above. In addition, we explicitly incorporate the prior sentiment of the argument and observe that this information helps reduce fitting errors.

\footnotetext{
${ }^{1}$ The sentiment values have been linearly rescaled from the original range $[0,1]$ to $[-0.5,0.5]$; in the figure a negative or positive value corresponds to a negative or a positive sentiment respectively; zero means neutral. The negator list will be discussed later in the paper.

${ }^{2}$ Similar distribution is observed in other data such as Tweets (Kiritchenko et al., 2014).
}

\section{Related work}

Automatic sentiment analysis The expression of sentiment is an integral component of human language. In written text, sentiment is conveyed with word senses and their composition, and in speech also via prosody such as pitch (Mairesse et al., 2012). Early work on automatic sentiment analysis includes the widely cited work of (Hatzivassiloglou and McKeown, 1997; Pang et al., 2002; Turney, 2002), among others. Since then, there has been an explosion of research addressing various aspects of the problem, including detecting subjectivity, rating and classifying sentiment, labeling sentiment-related semantic roles (e.g., target of sentiment), and visualizing sentiment (see surveys by Pang and Lee (2008) and Liu and Zhang (2012)).

Negation modeling Negation is a general grammatical category pertaining to the changing of the truth values of propositions; negation modeling is not limited to sentiment. For example, paraphrase and contradiction detection systems rely on detecting negated expressions and opposites (Harabagiu et al., 2006). In general, a negated expression and the opposite of the expression may or may not convey the same meaning. For example, not alive has the same meaning as dead, however, not tall does not always mean short. Some automatic methods to detect opposites were proposed by Hatzivassiloglou and McKeown (1997) and Mohammad et al. (2013).

Negation modeling for sentiment An early yet influential reversing assumption conjectures that a negator reverses the sign of the sentiment value of the modified text (Polanyi and Zaenen, 2004; Kennedy and Inkpen, 2006), e.g., from +0.5 to 0.5 , or vice versa. A different hypothesis, called the shifting hypothesis in this paper, assumes that negators change the sentiment values by a constant amount (Taboada et al., 2011; Liu and Seneff, 2009). Other approaches to negation modeling have been discussed in (Jia et al., 2009; Wiegand et al., 2010; Lapponi et al., 2012; Benamara et al., 2012).

In the process of semantic composition, the effect of negators could depend on the syntax and semantics of the text spans they modify. The approaches of modeling this include bag-of-wordbased models. For example, in the work of (Kennedy and Inkpen, 2006), a feature not_good will be created if the word good is encountered 
within a predefined range after a negator.

There exist different ways of incorporating more complicated syntactic and semantic information. Much recent work considers sentiment analysis from a semantic-composition perspective (Moilanen and Pulman, 2007; Choi and Cardie, 2008; Socher et al., 2012; Socher et al., 2013), which achieved the state-of-the-art performance. Moilanen and Pulman (2007) used a collection of hand-written compositional rules to assign sentiment values to different granularities of text spans. Choi and Cardie (2008) proposed a learning-based framework. The more recent work of (Socher et al., 2012; Socher et al., 2013) proposed models based on recursive neural networks that do not rely on any heuristic rules. Such models work in a bottom-up fashion over the parse tree of a sentence to infer the sentiment label of the sentence as a composition of the sentiment expressed by its constituting parts. The approach leverages a principled method, the forward and backward propagation, to learn a vector representation to optimize the system performance. In principle neural network is able to fit very complicated functions (Mitchell, 1997), and in this paper, we adapt the state-of-the-art approach described in (Socher et al., 2013) to help understand the behavior of negators specifically.

\section{Negation models based on heuristics}

We begin with previously proposed methods that leverage heuristics to model the behavior of negators. We then propose to extend them to consider lexical information of the negators themselves.

\subsection{Non-lexicalized assumptions and modeling}

In previous research, some influential, widely adopted assumptions posit the effect of negators to be independent of both the specific negators and the semantics and syntax of the arguments. In this paper, we call a model based on such assumptions a non-lexicalized model. In general, we can simply define this category of models in Equation 1. That is, the model parameters are only based on the sentiment value of the arguments.

$$
s\left(w_{n}, \vec{w}\right) \stackrel{\text { def }}{=} f(s(\vec{w}))
$$

\subsubsection{Reversing hypothesis}

A typical model falling into this category is the reversing hypothesis discussed in Section 2, where a negator simply reverses the sentiment score $s(\vec{w})$ to be $-s(\vec{w})$; i.e., $f(s(\vec{w}))=-s(\vec{w})$.

\subsubsection{Shifting hypothesis}

Basic shifting Similarly, a shifting based model depends on $s(\vec{w})$ only, which can be written as:

$$
f(s(\vec{w}))=s(\vec{w})-\operatorname{sign}(s(\vec{w})) * C
$$

where $\operatorname{sign}($.$) is the standard sign function$ which determines if the constant $C$ should be added to or deducted from $s\left(w_{n}\right)$ : the constant is added to a negative $s(\vec{w})$ but deducted from a positive one.

Polarity-based shifting As will be shown in our experiments, negators can have different shifting power when modifying a positive or a negative phrase. Thus, we explore the use of two different constants for these two situations, i.e., $f(s(\vec{w}))=$ $s(\vec{w})-\operatorname{sign}(s(\vec{w})) * C(\operatorname{sign}(s(\vec{w})))$. The constant $C$ now can take one of two possible values. We will show that this simple modification improves the fitting performance statistically significantly. Note also that instead of determining these constants by human intuition, we use the training data to find the constants in all shifting-based models as well as for the parameters in other models.

\subsection{Simple lexicalized assumptions}

The above negation hypotheses rely on $s(\vec{w})$. As intuitively shown in Figure 1, the capability of the non-lexicalized heuristics might be limited. Further semantic or syntactic information from either the negators or the phrases they modify could be helpful. The most straightforward way of expanding the non-lexicalized heuristics is probably to make the models to be dependent on the negators.

$$
s\left(w_{n}, \vec{w}\right) \stackrel{\text { def }}{=} f\left(w_{n}, s(\vec{w})\right)
$$

Negator-based shifting We can simply extend the basic shifting model above to consider the lexical information of negators: $f(s(\vec{w}))=s(\vec{w})-$ $\operatorname{sign}(s(\vec{w})) * C\left(w_{n}\right)$. That is, each negator has its own $C$. We call this model negator-based shifting. We will show that this model also statistically significantly outperforms the basic shifting without overfitting, although the number of parameters have increased. 
Combined shifting We further combine the negator-based shifting and polarity-based shifting above: $f(s(\vec{w}))=s(\vec{w})-\operatorname{sign}(s(\vec{w})) *$ $C\left(w_{n}, \operatorname{sign}(s(\vec{w}))\right)$. This shifting model is based on negators and the polarity of the text they modify: constants can be different for each negator-polarity pair. The number of parameters in this model is the multiplication of number of negators by two (the number of sentiment polarities). This model further improves the fitting performance on the test data.

\section{Semantics-enriched modeling}

Negators can interact with arguments in complex ways. Figure 1 shows the distribution of the effect of negators on sentiment without considering further semantics of the arguments. The question then is that whether and how much incorporating further syntax and semantic information can help better fit or predict the negation effect. Above, we have considered the semantics of the negators. Below, we further make the models to be dependent on the arguments. This can be written as:

$$
s\left(w_{n}, \vec{w}\right) \stackrel{\text { def }}{=} f\left(w_{n}, s(\vec{w}), r(\vec{w})\right)
$$

In the formula, $r(\vec{w})$ is a certain type of representation for the argument $\vec{w}$ and it models the semantics or/and syntax of the argument. There exist different ways of implementing $r(\vec{w})$. We consider two models in this study: one drops $s(\vec{w})$ in Equation 4 and directly models $f\left(w_{n}, r(\vec{w})\right)$. That is, the non-uniform information shown in Figure 1 is not directly modeled. The other takes into account $s(\vec{w})$ too.

For the former, we adopt the recursive neural tensor network (RNTN) proposed recently by Socher et al. (2013), which has showed to achieve the state-of-the-art performance in sentiment analysis. For the latter, we propose a prior sentimentenriched tensor network (PSTN) to take into account the prior sentiment of the argument $s(\vec{w})$.

\subsection{RNTN: Recursive neural tensor network}

A recursive neural tensor network (RNTN) is a specific form of feed-forward neural network based on syntactic (phrasal-structure) parse tree to conduct compositional sentiment analysis. For completeness, we briefly review it here. More details can be found in (Socher et al., 2013).

As shown in the black portion of Figure 2, each instance of RNTN corresponds to a binary parse

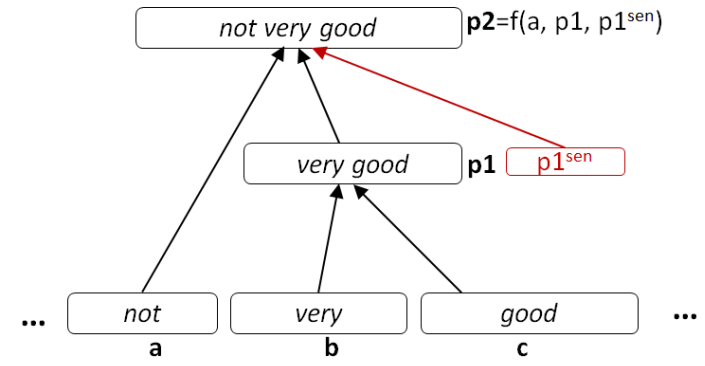

Figure 2: Prior sentiment-enriched tensor network (PSTN) model for sentiment analysis.

tree of a given sentence. Each node of the parse tree is a fixed-length vector that encodes compositional semantics and syntax, which can be used to predict the sentiment of this node. The vector of a node, say $p_{2}$ in Figure 2, is computed from the $d$ dimensional vectors of its two children, namely $a$ and $p_{1}\left(a, p_{1} \in \mathbb{R}^{d \times 1}\right)$, with a non-linear function:

$$
p_{2}=\tanh \left(\left[\begin{array}{c}
a \\
p_{1}
\end{array}\right]^{T} V^{[1: d]}\left[\begin{array}{c}
a \\
p_{1}
\end{array}\right]+W\left[\begin{array}{c}
a \\
p_{1}
\end{array}\right]\right)
$$

where, $W \in \mathbb{R}^{d \times(d+d)}$ and $V \in \mathbb{R}^{(d+d) \times(d+d) \times d}$ are the matrix and tensor for the composition function. A major difference of RNTN from the conventional recursive neural network (RRN) (Socher et al., 2012) is the use of the tensor $V$ in order to directly capture the multiplicative interaction of two input vectors, although the matrix $W$ implicitly captures the nonlinear interaction between the input vectors. The training of RNTN uses conventional forward-backward propagation.

\subsection{PSTN: Prior sentiment-enriched tensor network}

The non-uniform distribution in Figure 1 has showed certain correlations between the sentiment values of $s\left(w_{n}, \vec{w}\right)$ and $s(\vec{w})$, and such information has been leveraged in the models discussed in Section 3. We intend to devise a model that implements Equation 4. It bridges between the models we have discussed above that use either $s(\vec{w})$ or $r(\vec{w})$.

We extend RNTN to directly consider the sentiment information of arguments. Consider the node $p_{2}$ in Figure 2. When calculating its vector, we aim to directly engage the sentiment information of its right child, i.e., the argument. To this end, we make use of the sentiment class information of 
$p_{1}$, noted as $p_{1}^{\text {sen }}$. As a result, the vector of $p_{2}$ is calculated as follows:

$$
\begin{aligned}
p_{2} & =\tanh \left(\left[\begin{array}{c}
a \\
p_{1}
\end{array}\right]^{T} V^{[1: d]}\left[\begin{array}{c}
a \\
p_{1}
\end{array}\right]+W\left[\begin{array}{c}
a \\
p_{1}
\end{array}\right]\right. \\
& \left.+\left[\begin{array}{c}
a \\
p_{1}^{\text {sen }}
\end{array}\right]^{T} V^{\text {sen }^{[1: d]}}\left[\begin{array}{c}
a \\
p_{1}^{\text {sen }}
\end{array}\right]+W^{\text {sen }}\left[\begin{array}{c}
a \\
p_{1}^{\text {sen }}
\end{array}\right]\right)
\end{aligned}
$$

As shown in Equation 6, for the node vector $p_{1} \in \mathbb{R}^{d \times 1}$, we employ a matrix, namely $W^{\text {sen }} \in$ $\mathbb{R}^{d \times(d+m)}$ and a tensor, $V^{\text {sen }} \in \mathbb{R}^{(d+m) \times(d+m) \times d}$, aiming at explicitly capturing the interplays between the sentiment class of $p_{1}$, denoted as $p_{1}^{\text {sen }}(\in$ $\mathbb{R}^{m \times 1}$ ), and the negator $a$. Here, we assume the sentiment task has $m$ classes. Following the idea of Wilson et al. (2005), we regard the sentiment of $p_{1}$ as a prior sentiment as it has not been affected by the specific context (negators), so we denote our method as prior sentiment-enriched tensor network (PSTN). In Figure 2, the red portion shows the added components of PSTN.

Note that depending on different purposes, $p_{1}^{s e n}$ can take the value of the automatically predicted sentiment distribution obtained in forward propagation, the gold sentiment annotation of node $p_{1}$, or even other normalized prior sentiment value or confidence score from external sources (e.g., sentiment lexicons or external training data). This is actually an interesting place to extend the current recursive neural network to consider extrinsic knowledge. However, in our current study, we focus on exploring the behavior of negators. As we have discussed above, we will use the human annotated sentiment for the arguments, same as in the models discussed in Section 3.

With the new matrix and tensor, we then have $\theta=\left(V, V^{\text {sen }}, W, W^{\text {sen }}, W^{\text {label }}, L\right)$ as the PSTN model's parameters. Here, $L$ denotes the vector representations of the word dictionary.

\subsubsection{Inference and Learning}

Inference and learning in PSTN follow a forwardbackward propagation process similar to that in (Socher et al., 2013), and for completeness, we depict the details as follows. To train the model, one first needs to calculate the predicted sentiment distribution for each node:

$$
p_{i}^{\text {sen }}=W^{\text {label }} p_{i}, \quad p_{i}^{\text {sen }} \in \mathbb{R}^{m \times 1}
$$

and then compute the posterior probability over the $m$ labels:

$$
y^{i}=\operatorname{softmax}\left(p_{i}^{\text {sen }}\right)
$$

During learning, following the method used by the RNTN model in (Socher et al., 2013), PSTN also aims to minimize the cross-entropy error between the predicted distribution $y^{i} \in \mathbb{R}^{m \times 1}$ at node $i$ and the target distribution $t^{i} \in \mathbb{R}^{m \times 1}$ at that node. That is, the error for a sentence is calculated as:

$$
E(\theta)=\sum_{i} \sum_{j} t_{j}^{i} \log y_{j}^{i}+\lambda\|\theta\|^{2}
$$

where, $\lambda$ represents the regularization hyperparameters, and $j \in m$ denotes the $j$-th element of the multinomial target distribution.

To minimize $E(\theta)$, the gradient of the objective function with respect to each of the parameters in $\theta$ is calculated efficiently via backpropagation through structure, as proposed by Goller and Kchler (1996). Specifically, we first compute the prediction errors in all tree nodes bottom-up. After this forward process, we then calculate the derivatives of the softmax classifiers at each node in the tree in a top-down fashion. We will discuss the gradient computation for the $V^{s e n}$ and $W^{\text {sen }}$ in detail next. Note that the gradient calculations for the $V, W, W^{\text {label }}, L$ are the same as that of presented in (Socher et al., 2013).

In the backpropogation process of the training, each node (except the root node) in the tree carries two kinds of errors: the local softmax error and the error passing down from its parent node. During the derivative computation, the two errors will be summed up as the complement incoming error for the node. We denote the complete incoming error and the softmax error vector for node $i$ as $\delta^{i, c o m} \in \mathbb{R}^{d \times 1}$ and $\delta^{i, s} \in \mathbb{R}^{d \times 1}$, respectively. With this notation, the error for the root node $p_{2}$ can be formulated as follows.

$$
\begin{aligned}
\delta^{p_{2}, c o m} & =\delta^{p_{2}, s} \\
& =\left(W^{T}\left(y^{p_{2}}-t^{p_{2}}\right)\right) \otimes f^{\prime}\left(\left[a ; p_{1}\right]\right)
\end{aligned}
$$

where $\otimes$ is the Hadamard product between the two vectors and $f^{\prime}$ is the element-wise derivative of $f=\tanh$. With the results from Equation 8, we then can calculate the derivatives for the $W^{\text {sen }}$ at node $p_{2}$ using the following equation:

$$
\frac{\partial E^{p_{2}}}{W^{s e n}}=\delta^{p_{2}, c o m}\left(\left[a ; p_{1}^{s e n}\right]\right)^{T}
$$

Similarly, for the derivative of each slice $k(k=$ 
$1, \ldots, d)$ of the $V^{\text {sen }}$ tensor, we have the following:

$$
\frac{\partial E^{p_{2}}}{V_{[k]}^{s e n}}=\delta_{k}^{p_{2}, c o m}\left[\begin{array}{c}
a \\
p_{1}^{s e n}
\end{array}\right]\left[\begin{array}{c}
a \\
p_{1}^{s e n}
\end{array}\right]^{T}
$$

Now, let's form the equations for computing the error for the two children of the $p_{2}$ node. The difference for the error at $p_{2}$ and its two children is that the error for the latter will need to compute the error message passing down from $p_{2}$. We denote the error passing down as $\delta^{p_{2}, \text { down }}$, where the left child and the right child of $p_{2}$ take the $1^{\text {st }}$ and $2^{\text {nd }}$ half of the error $\delta^{p_{2}, \text { down }}$, namely $\delta^{p_{2}, \text { down }}[1: d]$ and $\delta^{p_{2}, \text { down }}[d+1: 2 d]$, respectively. Following this notation, we have the error message for the two children of $p_{2}$, provided that we have the $\delta^{p_{2}, \text { down }}$

$$
\begin{aligned}
\delta^{p_{1}, \text { com }} & =\delta^{p_{1}, s}+\delta^{p_{2}, d o w n}[d+1: 2 d] \\
& =\left(W^{T}\left(y^{p_{1}}-t^{p_{1}}\right)\right) \otimes f^{\prime}([b ; c]) \\
& +\delta^{p_{2}, d o w n}[d+1: 2 d]
\end{aligned}
$$

The incoming error message of node $a$ can be calculated similarly. Finally, we can finish the above equations with the following formula for computing $\delta^{p_{2}, d o w n}$ :

$$
\delta^{p_{2}, \text { down }}=\left(W^{T} \delta^{p_{2}, c o m}\right) \otimes f^{\prime}\left(\left[a ; p_{1}\right]\right)+\delta^{\text {tensor }}
$$

where

$$
\begin{aligned}
& \delta^{\text {tensor }}=\left[\delta^{V}[1: d]+\delta^{V^{s e n}}[1: d], \delta^{V}[d+1: 2 d]\right] \\
& =\sum_{k=1}^{d} \delta_{k}^{p_{2}, c o m}\left(V_{[k]}+\left(V_{[k]}\right)^{T}\right) \otimes f^{\prime}\left(\left[a ; p_{1}\right]\right)[1: d] \\
& +\sum_{k=1}^{d} \delta_{k}^{p_{2}, c o m}\left(V_{[k]}^{s e n}+\left(V_{[k]}^{s e n}\right)^{T}\right) \otimes f^{\prime}\left(\left[a ; p_{1}^{s e n}\right]\right)[1: d] \\
& +\sum_{k=1}^{d} \delta_{k}^{p_{2}, c o m}\left(V_{[k]}+\left(V_{[k]}\right)^{T}\right) \otimes f^{\prime}\left(\left[a ; p_{1}\right]\right)[d+1: 2 d]
\end{aligned}
$$

After the models are trained, they are applied to predict the sentiment of the test data. The original RNTN and the PSTN predict 5-class sentiment for each negated phrase; we map the output to real-valued scores based on the scale that Socher et al. (2013) used to map real-valued sentiment scores to sentiment categories. Specifically, we conduct the mapping with the formula: $p_{i}^{\text {real }}=$ $y^{i} \cdot\left[\begin{array}{lllll}0.1 & 0.3 & 0.5 & 0.7 & 0.9\end{array}\right]$; i.e., we calculate the dot product of the posterior probability $y^{i}$ and the scaling vector. For example, if $y^{i}=\left[\begin{array}{lllll}0.5 & 0.5 & 0 & 0 & 0\end{array}\right]$, meaning this phrase has a 0.5 probability to be in the first category (strong negative) and 0.5 for the second category (weak negative), the resulting $p_{i}^{\text {real }}$ will be $0.2\left(0.5^{*} 0.1+0.5^{*} 0.3\right)$.

\section{Experiment set-up}

Data As described earlier, the Stanford Sentiment Treebank (Socher et al., 2013) has manually annotated, real-valued sentiment values for all phrases in parse trees. This provides us with the training and evaluation data to study the effect of negators with syntax and semantics of different complexity in a natural setting. The data contain around 11,800 sentences from movie reviews that were originally collected by Pang and Lee (2005). The sentences were parsed with the Stanford parser (Klein and Manning, 2003). The phrases at all tree nodes were manually annotated with one of 25 sentiment values that uniformly span between the positive and negative poles. The values are normalized to the range of $[0,1]$.

In this paper, we use a list of most frequent negators that include the words not, no, never, and their combinations with auxiliaries (e.g., didn't). We search these negators in the Stanford Sentiment Treebank and normalize the same negators to a single form; e.g., "is n't", "isn't", and "is not" are all normalized to "is_not". Each occurrence of a negator and the phrase it is directly composed with in the treebank, i.e., $\left\langle w_{n}, \vec{w}\right\rangle$, is considered a data point in our study. In total, we collected 2,261 pairs, including 1,845 training and 416 test cases. The split of training and test data is same as specified in (Socher et al., 2013).

Evaluation metrics We use the mean absolute error (MAE) to evaluate the models, which measures the averaged absolute offsets between the predicted sentiment values and the gold standard. More specifically, MAE is calculated as: $M A E=\frac{1}{N} \sum_{\left\langle w_{n}, \vec{w}\right\rangle}\left|\left(\hat{s}\left(w_{n}, \vec{w}\right)-s\left(w_{n}, \vec{w}\right)\right)\right|$, where $\hat{s}\left(w_{n}, \vec{w}\right)$ denotes the gold sentiment value and $s\left(w_{n}, \vec{w}\right)$ the predicted one for the pair $\left\langle w_{n}, \vec{w}\right\rangle$, and $\mathrm{N}$ is the total number of test instances. Note that mean square error (MSE) is another widely used measure for regression, but it is less intuitive for out task here.

\section{Experimental results}

Overall regression performance Table 1 shows the overall fitting performance of all models. The first row of the table is a random baseline, which 
simply guesses the sentiment value for each test case randomly in the range [0,1]. The table shows that the basic reversing and shifting heuristics do capture negators' behavior to some degree, as their MAE scores are higher than that of the baseline. Making the basic shifting model to be dependent on the negators (model 4) reduces the prediction error significantly as compared with the error of the basic shifting (model 3 ). The same is true for the polarity-based shifting (model 5), reflecting that the roles of negators are different when modifying positive and negative phrases. Merging these two models yields additional improvement (model 6).

\begin{tabular}{ll}
\hline Assumptions & MAE \\
\hline Baseline & \\
$\quad$ (1) Random & 0.2796 \\
Non-lexicalized & \\
$\quad$ (2) Reversing & $0.1480^{*}$ \\
$\quad$ (3) Basic shifting & $0.1452^{*}$ \\
Simple-lexicalized & \\
(4) Negator-based shifting & $0.1415 \dagger$ \\
(5) Polarity-based shifting & $0.1417 \dagger$ \\
(6) Combined shifting & $0.1387 \dagger$ \\
Semantics-enriched & \\
(7) RNTN & $0.1097^{* *}$ \\
(8) PSTN & $0.1062 \dagger \dagger$ \\
\hline
\end{tabular}

Table 1: Mean absolute errors (MAE) of fitting different models to Stanford Sentiment Treebank. Models marked with an asterisk $(*)$ are statistically significantly better than the random baseline. Models with a dagger sign ( $\dagger$ ) significantly outperform model (3). Double asterisks ** indicates a statistically significantly different from model (6), and the model with the double dagger $\dagger \dagger$ is significantly better than model (7). One-tailed paired t-test with a 95\% significance level is used here.

Furthermore, modeling the syntax and semantics with the state-of-the-art recursive neural network (model 7 and 8) can dramatically improve the performance over model 6. The PSTN model, which takes into account the human-annotated prior sentiment of arguments, performs the best. This could suggest that additional external knowledge, e.g., that from human-built resources or automatically learned from other data (e.g., as in (Kiritchenko et al., 2014)), including sentiment that cannot be inferred from its constituent expressions, might be incorporated to benefit the current

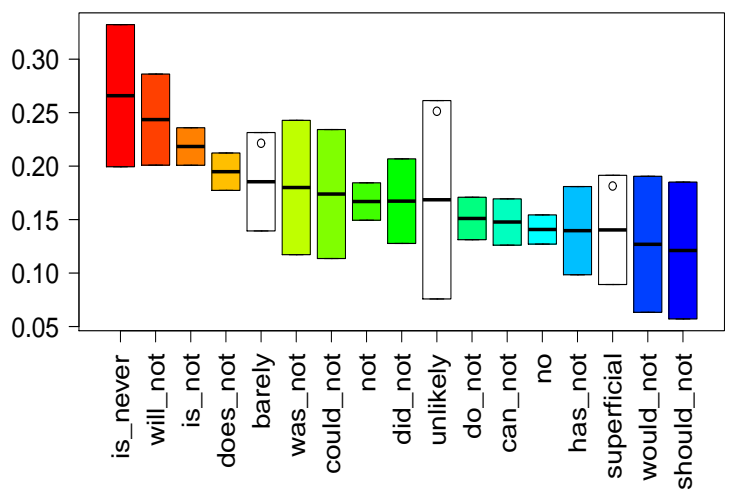

Figure 3: Effect of different negators in shifting sentiment values.

neural-network-based models as prior knowledge.

Note that the two neural network based models incorporate the syntax and semantics by representing each node with a vector. One may consider that a straightforward way of considering the semantics of the modified phrases is simply memorizing them. For example, if a phrase very good modified by a negator not appears in the training and test data, the system can simply memorize the sentiment score of not very good in training and use this score at testing. When incorporating this memorizing strategy into model (6), we observed a MAE score of 0.1222 . It's not surprising that memorizing the phrases has some benefit, but such matching relies on the exact reoccurrences of phrases. Note that this is a special case of what the neural network based models can model.

Discriminating negators The results in Table 1 has demonstrated the benefit of discriminating negators. To understand this further, we plot in Figure 3 the behavior of different negators: the $\mathrm{x}$-axis is a subset of our negators and the y-axis denotes absolute shifting in sentiment values. For example, we can see that the negator "is_never" on average shifts the sentiment of the arguments by 0.26 , which is a significant change considering the range of sentiment value is $[0,1]$. For each negator, a 95\% confidence interval is shown by the boxes in the figure, which is calculated with the bootstrapping resampling method. We can observe statistically significant differences of shifting abilities between many negator pairs such as that between "is_never" and "do_not" as well as between "does_not" and "can_not".

Figure 3 also includes three diminishers (the 


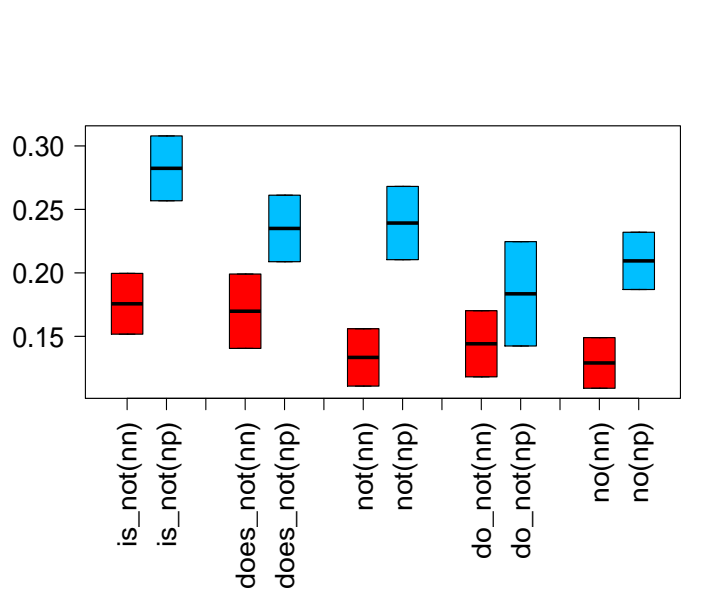

Figure 4: The behavior of individual negators in negated negative (nn) and negated positive (np) context.

white bars), i.e., barely, unlikely, and superficial. By following (Kennedy and Inkpen, 2006), we extracted 319 diminishers (also called understatement or downtoners) from General Inquirer ${ }^{3}$. We calculated their shifting power in the same manner as for the negators and found three diminishers having shifting capability in the shifting range of these negators. This shows that the boundary between negators and diminishers can by fuzzy. In general, we argue that one should always consider modeling negators individually in a sentiment analysis system. Alternatively, if the modeling has to be done in groups, one should consider clustering valence shifters by their shifting abilities in training or external data.

Figure 4 shows the shifting capacity of negators when they modify positive (blue boxes) or negative phrases (red boxes). The figure includes five most frequently used negators found in the sentiment treebank. Four of them have significantly different shifting power when composed with positive or negative phrases, which can explain why the polarity-based shifting model achieves improvement over the basic shifting model.

Modeling syntax and semantics We have seen above that modeling syntax and semantics through the-state-of-the-art neural networks help improve the fitting performance. Below, we take a closer look at the fitting errors made at different depths of the sentiment treebank. The depth here is defined as the longest distance between the root of a negator-phrase pair $\left\langle w_{n}, \vec{w}\right\rangle$ and their descendant

\footnotetext{
${ }^{3}$ http://www.wjh.harvard.edu/ inquirer/
}

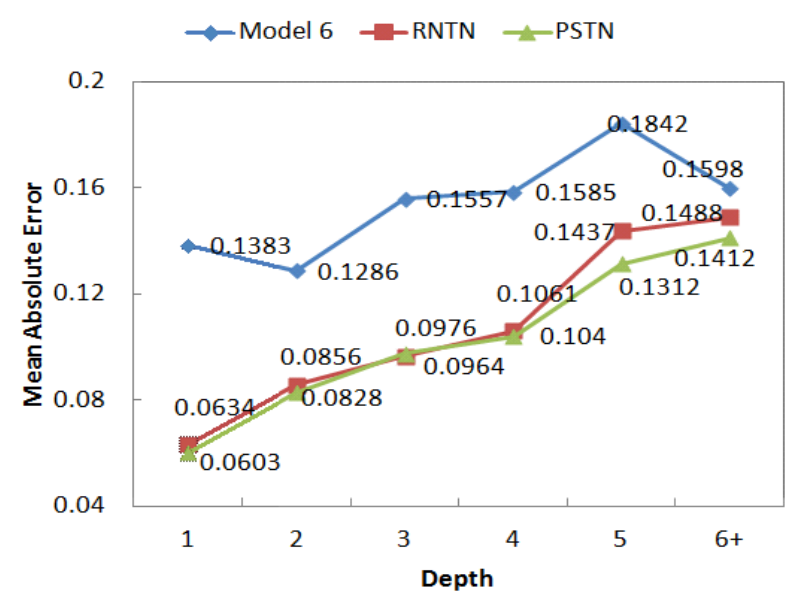

Figure 5: Errors made at different depths in the sentiment tree bank.

leafs. Negators appearing at deeper levels of the tree tend to have more complicated syntax and semantics. In Figure 5, the x-axis corresponds to different depths and y-axis is the mean absolute errors (MAE).

The figure shows that both RNTN and PSTN perform much better at all depths than the model 6 in Table 1. When the depths are within 4, the RNTN performs very well and the (human annotated) prior sentiment of arguments used in PSTN does not bring additional improvement over RNTN. PSTN outperforms RNTN at greater depths, where the syntax and semantics are more complicated and harder to model. The errors made by model 6 is bumpy, as the model considers no semantics and hence its errors are not dependent on the depths. On the other hand, the errors of RNTN and PSTN monotonically increase with depths, indicating the increase in the task difficulty.

\section{Conclusions}

Negation plays a fundamental role in modifying sentiment. In the process of semantic composition, the impact of negators is complicated by the syntax and semantics of the text spans they modify. This paper provides a comprehensive and quantitative study of the behavior of negators through a unified view of fitting human annotation. We first measure the modeling capabilities of two influential heuristics on a sentiment treebank and find that they capture some effect of negation; however, extending these non-lexicalized models to be dependent on the negators improves the per- 
formance statistically significantly. The detailed analysis reveals the differences in the behavior among negators, and we argue that they should always be modeled separately. We further make the models to be dependent on the text being modified by negators, through adaptation of a state-ofthe-art recursive neural network to incorporate the syntax and semantics of the arguments; we discover this further reduces fitting errors.

\section{References}

Farah Benamara, Baptiste Chardon, Yannick Mathieu, Vladimir Popescu, and Nicholas Asher. 2012. How do negation and modality impact on opinions? In Proceedings of the ACL-2012 Workshop on ExtraPropositional Aspects of Meaning in Computational Linguistics, pages 10-18, Jeju, Republic of Korea.

Yejin Choi and Claire Cardie. 2008. Learning with compositional semantics as structural inference for subsentential sentiment analysis. In Proceedings of the Conference on Empirical Methods in Natural Language Processing, EMNLP '08, pages 793-801, Honolulu, Hawaii.

Christoph Goller and Andreas Kchler. 1996. Learning task-dependent distributed representations by backpropagation through structure. In In Proc. of the ICNN-96, pages 347-352, Bochum, Germany. IEEE.

Sanda Harabagiu, Andrew Hickl, and Finley Lacatusu. 2006. Negation, contrast and contradiction in text processing. In $A A A I$, volume 6, pages 755-762.

Vasileios Hatzivassiloglou and Kathleen R. McKeown. 1997. Predicting the semantic orientation of adjectives. In Proceedings of the 8th Conference of European Chapter of the Association for Computational Linguistics, EACL '97, pages 174-181, Madrid, Spain.

Lifeng Jia, Clement Yu, and Weiyi Meng. 2009. The effect of negation on sentiment analysis and retrieval effectiveness. In Proceedings of the 18th ACM Conference on Information and Knowledge Management, CIKM '09, pages 1827-1830, Hong Kong, China. ACM.

Alistair Kennedy and Diana Inkpen. 2006. Sentiment classification of movie reviews using contextual valence shifters. Computational Intelligence, 22(2):110-125.

Svetlana Kiritchenko, Xiaodan Zhu, and Saif Mohammad. 2014. Sentiment analysis of short informal texts. (to appear) Journal of Artificial Intelligence Research.

Dan Klein and Christopher D. Manning. 2003. Accurate unlexicalized parsing. In Proceedings of the 41st Annual Meeting on Association for Computational Linguistics - Volume 1, ACL '03, pages 423430, Sapporo, Japan. Association for Computational Linguistics.

Emanuele Lapponi, Jonathon Read, and Lilja Ovrelid. 2012. Representing and resolving negation for sentiment analysis. In Jilles Vreeken, Charles Ling, Mohammed Javeed Zaki, Arno Siebes, Jeffrey $\mathrm{Xu} \mathrm{Yu,} \mathrm{Bart} \mathrm{Goethals,} \mathrm{Geoffrey} \mathrm{I.} \mathrm{Webb,} \mathrm{and}$ Xindong Wu, editors, ICDM Workshops, pages 687692. IEEE Computer Society.

Jingjing Liu and Stephanie Seneff. 2009. Review sentiment scoring via a parse-and-paraphrase paradigm. In $E M N L P$, pages 161-169, Singapore.

Bing Liu and Lei Zhang. 2012. A survey of opinion mining and sentiment analysis. In Charu C. Aggarwal and ChengXiang Zhai, editors, Mining Text Data, pages 415-463. Springer US.

François Mairesse, Joseph Polifroni, and Giuseppe Di Fabbrizio. 2012. Can prosody inform sentiment analysis? experiments on short spoken reviews. In ICASSP, pages 5093-5096, Kyoto, Japan.

Tom M Mitchell. 1997. Machine learning. 1997. Burr Ridge, IL: McGraw Hill, 45.

Saif M. Mohammad, Bonnie J. Dorr, Graeme Hirst, and Peter D. Turney. 2013. Computing lexical contrast. Computational Linguistics, 39(3):555-590.

Karo Moilanen and Stephen Pulman. 2007. Sentiment composition. In Proceedings of RANLP 2007, Borovets, Bulgaria.

Roser Morante and Caroline Sporleder. 2012. Modality and negation: An introduction to the special issue. Computational linguistics, 38(2):223-260.

Bo Pang and Lillian Lee. 2005. Seeing stars: Exploiting class relationships for sentiment categorization with respect to rating scales. In Proceedings of the Annual Meeting of the Association for Computational Linguistics, ACL '05, pages 115-124.

Bo Pang and Lillian Lee. 2008. Opinion mining and sentiment analysis. Foundations and Trends in Information Retrieval, 2(1-2):1-135.

Bo Pang, Lillian Lee, and Shivakumar Vaithyanathan. 2002. Thumbs up? sentiment classification using machine learning techniques. In Proceedings of EMNLP, pages 79-86, Philadelphia, USA.

Livia Polanyi and Annie Zaenen. 2004. Contextual valence shifters. In Exploring Attitude and Affect in Text: Theories and Applications (AAAI Spring Symposium Series).

Richard Socher, Brody Huval, Christopher D. Manning, and Andrew Y. Ng. 2012. Semantic compositionality through recursive matrix-vector spaces. In 
Proceedings of the Conference on Empirical Methods in Natural Language Processing, EMNLP '12, Jeju, Korea. Association for Computational Linguistics.

Richard Socher, Alex Perelygin, Jean Y. Wu, Jason Chuang, Christopher D. Manning, Andrew Y. Ng, and Christopher Potts. 2013. Recursive deep models for semantic compositionality over a sentiment treebank. In Proceedings of the Conference on Empirical Methods in Natural Language Processing, EMNLP' 13, Seattle, USA. Association for Computational Linguistics.

Maite Taboada, Julian Brooke, Milan Tofiloski, Kimberly Voll, and Manfred Stede. 2011. Lexiconbased methods for sentiment analysis. Computational Linguistics, 37(2):267-307.

Peter Turney. 2002. Thumbs up or thumbs down? semantic orientation applied to unsupervised classification of reviews. In ACL, pages 417-424, Philadelphia, USA.

Michael Wiegand, Alexandra Balahur, Benjamin Roth, Dietrich Klakow, and Andrés Montoyo. 2010. A survey on the role of negation in sentiment analysis. In Proceedings of the Workshop on Negation and Speculation in Natural Language Processing, NeSpNLP '10, pages 60-68, Stroudsburg, PA, USA. Association for Computational Linguistics.

Theresa Wilson, Janyce Wiebe, and Paul Hoffmann. 2005. Recognizing contextual polarity in phraselevel sentiment analysis. In Proceedings of the Conference on Human Language Technology and Empirical Methods in Natural Language Processing, HLT '05, pages 347-354, Stroudsburg, PA, USA. Association for Computational Linguistics. 